\title{
Overview of neutrino-nucleus quasielastic scattering
}

Cite as: AIP Conference Proceedings 1189, 125 (2009); https://doi.org/10.1063/1.3274142

Published Online: 02 December 2009

J. L. Herraiz, M. C. Martínez, J. A. Caballero, and J. M. Udías

\section{ARTICLES YOU MAY BE INTERESTED IN}

Theory and phenomenology of coherent neutrino-nucleus scattering

AIP Conference Proceedings 1666, 160001 (2015); https://doi.org/10.1063/1.4915590

Comparison of Models of Neutrino-Nucleus Interactions

AIP Conference Proceedings 1189, 60 (2009); https://doi.org/10.1063/1.3274191

Neutrino cross-sections: Experiments

AIP Conference Proceedings 1666, 060003 (2015); https://doi.org/10.1063/1.4915559
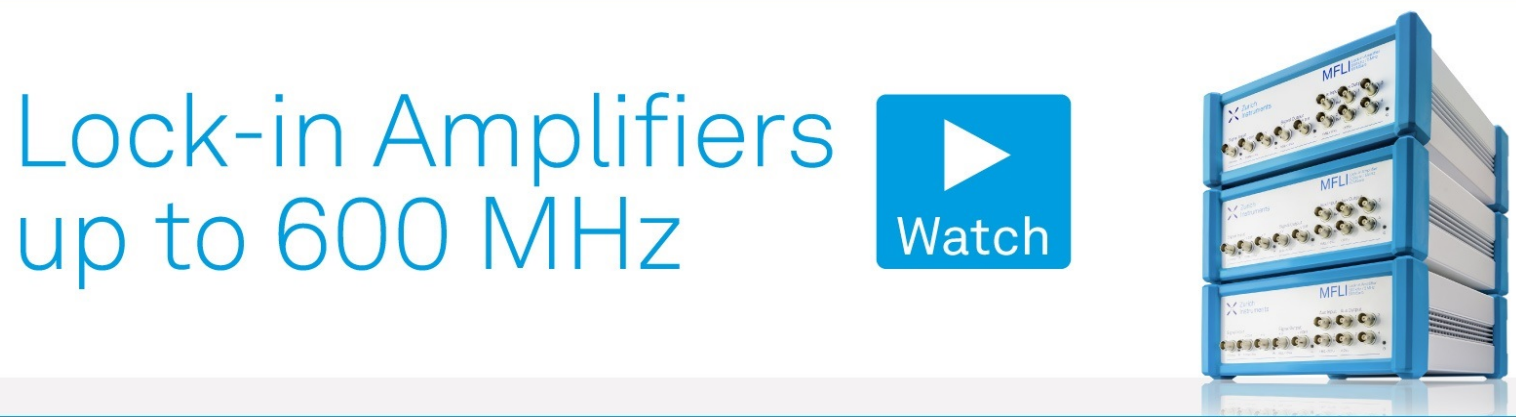


\title{
Overview of neutrino-nucleus quasielastic scattering
}

\author{
J.L. Herraiz*, M.C. Martínez*, J.A. Caballero ${ }^{\dagger}$ and J.M. Udías* \\ ${ }^{*}$ Grupo de Física Nuclear, Departamento de Física Atómica, Molecular y Nuclear \\ Universidad Complutense de Madrid, E-28040 Madrid, SPAIN \\ ${ }^{\dagger}$ Departamento de Física Atómica, Molecular y Nuclear \\ Universidad de Sevilla, Apdo. 1065, E-41080 Sevilla, SPAIN
}

\begin{abstract}
A review of quasielastic neutrino-nucleus scattering will be presented, with emphasis on bringing together the knowledge (and language) of neutrino physics, electron scattering, and nuclear structure communities. Assumptions commonly made which simplify the theoretical calculations will be examined. Finally, an attempt will be made to identify places where improvement from either theoretical or experimental sides would be more significant.
\end{abstract}

Keywords: lepton induced reactions, neutrino scattering, electron scattering, nuclear structure PACS: 24.10.Jv, 25.30.-c, 25.30.Fj, 25.30.Pt

\section{INTRODUCTION}

The presence of neutrinos, being chargeless particles, can only be inferred by detecting the secondary particles they create when colliding and interacting with matter. Nuclei are most often used as neutrino detectors, providing relatively large cross sections that offer a broad variety of information. Thus, accurate predictions for neutrino-nucleus cross sections are needed in the analyses of on-going and future experimental studies of neutrino reactions and neutrino oscillations [1,2,3] at intermediate energies, that is, energies beyond the nuclear resonance region. In this survey we focus in single-nucleon knockout without pion production, often referred to as quasi-elastic (QE) contribution to the inclusive neutrino-nucleus cross sections, for energies relevant to proposals like MINERvA [3], MiniBooNE [4] and FINeSSE [6].

Neutrino and electron scattering reactions are very similar in the theoretical treatment, even if from the experimental point of view, they may be quite different. For electron scattering, experiments are performed with monochromatic beams, while for neutrinos, beams are produced with finite size and sizeable span in energy. Moreover, the knowledge about these neutrino beams is somewhat indirect, based upon MC simulations. Surely after many years of research in this area, these beams are better known and validated against expected results for well known reactions (neutrinoelectron cross-sections), yet it is clear that neutrino beams are not as well under control as electron beams. This may change in the future with the availability of beta beam factories that would allow for an order of magnitude increase in the precise knowledge of neutrino beam properties.

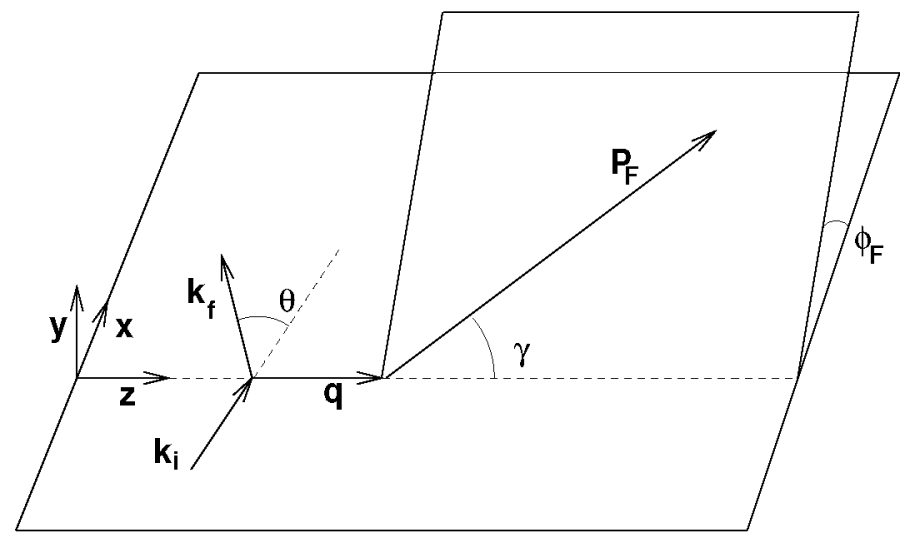

FIGURE 1. Kinematics of the lepton-nucleus processes considered in this work 

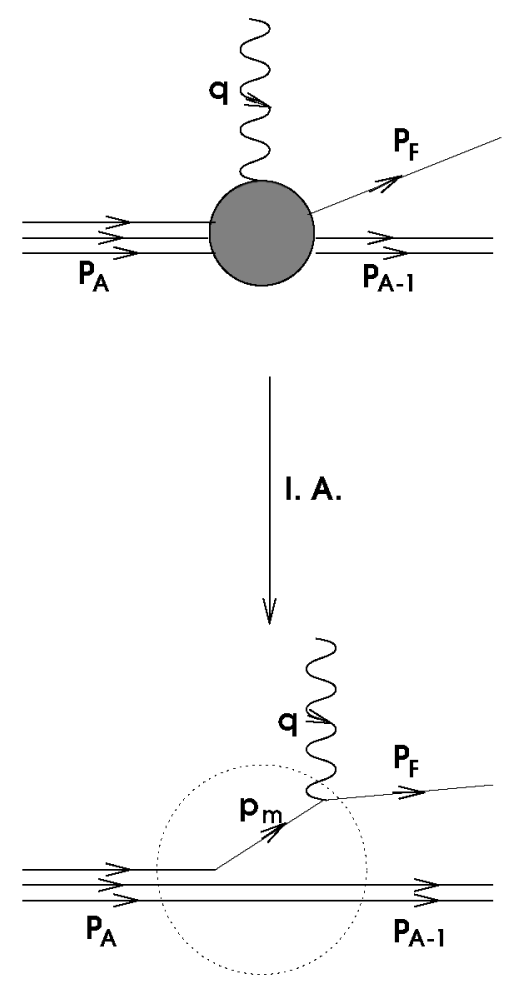

FIGURE 2. The lepton-nucleus reaction in first order Born Approximation (upper part of the figure) and within the Impulse Approximation (IA) picture (lower part of the figure)

Further, with electrons the scattered lepton can be detected, and even a knocked out nucleon can be measured in coincidence. Coincidence experiments extend the range of reactions that can be studied under exclusive conditions, by exclusive meaning that the channel measured is the only one that can contribute to the measurement, making this scenario ideally suited to test models against experiment. Indeed, the exclusive electron-nucleus experiments are extremely well described by theory. For electrons, inclusive experiments have more difficulty, and analysis of transparency data pose many difficulties for the theoretical models. While occupancies of the (valence) shells deduced from exclusive coincidence $\left(e, e^{\prime} p\right)$ experiments show the role of correlations causing the depletion of occupancies, this is not much considered when comparing inclusive experiments to similar models. While it is true that the missing nucleonic strength estimated from exclusive experiments may contribute to inclusive measurements at energy regions where the exclusive experiments are not looking at, it is also true that this redistribution of the strength due to correlations must have some effect on the inclusive cross-section. Further, deep (i.e., non valence) nucleon shells cannot be studied under exclusive conditions with the usual, nucleus at rest, experiments. Future $e-A$ colliders [7] will open the possibility of studying deep shells under exclusive conditions, which will add sets of data to validate theoretical models or to extract nuclear response to leptonic probes in regions unexplored so far.

The experience with electrons can be translated to neutrinos, in the case of charged current, the final lepton is often detected and its energy and/or direction is relatively well known, so that this reaction parallels electron-nucleus experiments. On the other hand, for neutral currents only the final nucleons may be detected and this has no parallel on electron experiments. Thus, the description of neutrino experiments would most likely rely on multi-channel calculations, where all processes that the lepton may induce are included, as well as all possible events that may happen to the nucleons. These calculations are very involved, but should become feasible in the near future with only modest approximations that will allow for incorporating them into MC event generators. As an example the GiBUU model [8], which reproduces adequately electron transparency data. It is of paramount importance in order to asses the reliability of the models employed in neutrino-nucleus scattering, to contrast them against electron observables, either exclusive, inclusive or partially inclusive/exclusive data, that have been measured or will become known in the near future. 


\section{LEPTON-NUCLEUS CROSS-SECTION}

\section{One boson exchange}

We will discuss neutrino interactions with nuclei with the language usually employed in electron-nucleus scattering. From the theoretical point of view, both projectiles can be dealt within the lowest order of approximation, that is, with the exchange of one intermediate boson (OBE), either a virtual photon or $W^{ \pm}$or $Z^{0}$. This implicitly assumes that the lepton can be well described as a free state, that is a plane wave, and thus this is sometimes referred as the 'Plane Wave Born approximation' or PWBA. The single-boson approximation allows for factorizing the leptonic variables at the lepton vertex from the one at the hadron vertex. We quote expressions (for details of the derivation see Refs. $[9,10]$ ) for neutrino and antineutrino neutral-current $(\mathrm{NC})$ and charged current $(\mathrm{CC})$ reactions, from nuclei which result in one emitted nucleon:

$$
\begin{gathered}
v(\bar{v})+A \Longrightarrow v(\bar{v})+N+(A-1) . \\
v(\bar{v})+A \Longrightarrow l(\bar{l})+N+(A-1) .
\end{gathered}
$$

$l$ labels the flavor of the lepton, and $A$ represents a nucleus with mass number $A$. In Figs. 1 and 2 our conventions for the kinematical variables are defined. The four-momenta of the incident neutrino and scattered lepton are labeled $k_{i}^{\mu}$ and $k_{f}^{\mu}$. Further, $P_{A}^{\mu}, P_{A-1}^{\mu}$ and $P_{F}^{\mu}$ represent the four-momenta of the target nucleus, the residual nucleus and the ejected nucleon. The $x y z$ coordinate system is chosen such that the $z$-axis lies along the momentum transfer $\vec{q}$, the $y$-axis along $\vec{k}_{i} \times \vec{k}_{f}$ and the $x$-axis lies in the scattering plane. The hadron reaction plane is then defined by $\vec{P}_{F}$ and $\vec{q}$. We adopt the standard convention $Q^{2} \equiv-q_{\mu} q^{\mu}$ for the four-momentum transfer.

Within the one boson exchange approximation and the extreme relativistic limit, the cross-section can be written as $[9,10,11]$ :

$$
\begin{aligned}
\frac{d^{5} \sigma}{d \varepsilon_{f} d^{2} \Omega d^{2} \Omega_{F}}= & \frac{M M_{A-1}}{(2 \pi)^{3} M_{A}} P_{F} f_{\text {rec }}^{-1} \sigma_{M}^{Z, W^{ \pm}} \\
& \times\left[v_{L} R_{L}+v_{T} R_{T}+v_{T T} R_{T T} \cos 2 \phi_{F}\right. \\
& \left.+v_{T L} R_{T L} \cos \phi_{F}+h\left(v_{T}^{\prime} R_{T}^{\prime}+v_{T L}^{\prime} R_{T L}^{\prime} \cos \phi_{F}\right)\right],
\end{aligned}
$$

with $\sigma_{M}$ defined by

$$
\sigma_{M}^{Z}=\left(\frac{G_{F} \cos (\theta / 2) \varepsilon_{f} M_{Z}^{2}}{\sqrt{2} \pi\left(Q^{2}+M_{Z}^{2}\right)}\right)^{2},
$$

for $\mathrm{NC}$ reactions and

$$
\sigma_{M}^{W^{ \pm}}=\sqrt{1-\frac{M_{l}^{2}}{\varepsilon_{f}^{2}}}\left(\frac{G_{F} \cos \left(\theta_{c}\right) \varepsilon_{f} M_{W}^{2}}{2 \pi\left(Q^{2}+M_{W}^{2}\right)}\right)^{2},
$$

for $\mathrm{CC}$ reactions. $M, M_{A}, M_{A-1}$ are the masses of the nucleon and the initial and final nuclei, $\theta_{c}$ is the Cabibbo angle and $h$ is the helicity of the lepton, $h=-1$ for neutrinos and $h=+1$ for antineutrinos.

For electron-nucleus scattering, a similar expression to Eq. (3) is obtained, factoring out the usual Mott cross-section for the scattering of a point-like electron by a point-like spin $1 / 2$ particle. This a direct consequence of the single boson exchange approximation, that also makes it possible to separate the dependence on the lepton kinematics contained in the $v$ factors from the nuclear part, contained in the responses $R$ (for explicit expressions of these see $[9,10]$ ).

Depending on the reaction considered, in order to obtain the QE neutrino-nucleus cross-section, one integrates over the phase space of the scattered lepton $\left(d^{2} \Omega\right)$ and/or the one of the outgoing nucleon $\left(d^{2} \Omega_{F}\left(\theta_{F}, \phi_{F}\right)\right)$. In this latter case, if integration over the azimuthal angle $\phi_{F}$ is complete, (as for instance if no attempt to detect the direction of the knocked-out nucleons is made) yields a factor $2 \pi$, whilst only the $\phi_{F}$-independent terms $\left(R_{L}, R_{T}, R_{T}^{\prime}\right)$ survive due to symmetry properties.

The determination of the nuclear response functions requires knowledge of the nuclear current matrix elements. To compute these, one further introduces the Impulse Approximation (IA), assuming that the incident neutrino interacts with only one nucleon, which is subsequently emitted. Under these assumptions, eventually (see next sections) the nuclear current is written as a sum of single-nucleon-like currents. The transition matrix elements can be cast in the following form:

$$
\left\langle J^{\mu}\right\rangle=\int d \vec{r} \bar{\Psi}_{F}(\vec{r}) \hat{J}^{\mu}(\vec{r}) e^{i \vec{q} \cdot \vec{r}} \Psi_{\alpha}(\vec{r}),
$$


where $\Psi_{\alpha}$ and $\Psi_{F}$ are respectively the overlap function between the initial and final nucleus and the scattering wave function for the knocked out nucleon. Further, $\hat{J}^{\mu}$ is the relativistic one-body current operator modeling the coupling between the virtual $Z^{0}$ or $W^{ \pm}$boson and a nucleon. In the spirit of the IA, a relativistic one-body vertex function is used:

$$
J^{\mu}=F_{1}\left(Q^{2}\right) \gamma^{\mu}+i \frac{\kappa}{2 M} F_{2}\left(Q^{2}\right) \sigma^{\mu v} q_{v}+G_{A}\left(Q^{2}\right) \gamma^{\mu} \gamma_{5}+\frac{1}{2 M} G_{P}\left(Q^{2}\right) q^{\mu} \gamma_{5},
$$

with $\kappa$ the anomalous magnetic moment. The weak vector form factors $F_{1}$ and $F_{2}$ can be related to the corresponding electromagnetic ones by the conserved vector current (CVC) hypothesis, details can be found in the literature [11]. The axial form factor for proton knockout is expressed as $G_{A}=-g_{A} / 2 \times G\left(Q^{2}\right)$ for NC reactions, $G_{A}=g_{A} \times G\left(Q^{2}\right)$ for $\mathrm{CC}$ reactions, and the same expressions with a - sign for neutrons. The experimental value taken for $g_{A}$ is 1.262 , and the axial form factor is usually assumed with a dipole form, $G\left(Q^{2}\right)=\left(1+Q^{2} / M_{A}^{2}\right)^{-2}$. The value of the axial mass $M_{A}$ is usually taken $1.032 \mathrm{GeV}$. The Goldberger-Treiman relation allows one to write the pseudoscalar form factor as

$$
G_{P}\left(Q^{2}\right)=\frac{2 M_{N}}{Q^{2}+m_{\pi}^{2}} G_{A}\left(Q^{2}\right),
$$

where $m_{\pi}$ denotes the pion mass. The contribution of this form factor, being proportional to the mass of the scattered lepton, vanishes for $\mathrm{NC}$ reactions.

The nuclear overlap function will be dealt with later in this work, here we will comment the following points:

- The vector form factors that enter in Eq. (7) are usually obtained from EM form factors measured in electron scattering experiments off free nucleons. These include two photon exchange contributions that should be removed or taken care of when applied to neutrino scattering. Polarization measurements of the EM form factors of the nucleons help in this regard.

- When applying Eq. (7) to off-shell nucleons in nucleus, we must be aware that some off-shell effects will remain in the calculation, and thus the form of the current operator depicted in Eq. (7) is not uniquely determined. Offshell effects are usually small for QE kinematics and they have not prevented extremely successful comparisons of theory to electron scattering data. However, one must be cautious, specially with regard to the axial term that is not conserved as the vector ones and thus can be seriously modified for off-shell nucleons with respect to free ones $[12,13,15,26,27]$.

- For NC scattering, strange quark contribution to the form factors can not be excluded, both to EM and axial form factors. Electron-nucleus parity violation (PV), as well as NC neutrino scattering in free nucleons have put stringent bounds on the strange form factors, and further electron PV experiments will make uncertainties due to the strangeness content of the nucleon even smaller. It is however worth mention that further experimental measurement of neutron-proton relative yields or asymmetries, or the so-called Wolfestein parameter, in neutrino experiments, will be very useful in settling down the contribution from strange form factors [19, 20, 21].

- While for CC scattering we do not have to worry about strangeness content, we must however realize that in this case a non negligible change in the mass of the particles involved in the lepton vertex may happen, for instance when muon neutrinos are involved. A vector $q^{\mu}$ term (a second class current) in Eq. (7) can have a non zero contribution in this case. However, for the usual range of QE kinematics, this would be a very small contribution, say a $1 \%$ effect. Rather than worrying about it, it is a good place to look for possible second class current contributions.

- The explicit separation of leptonic and hadronic variables, due to single boson exchange, allows for an independent computation of the hadronic responses, valid for all possible kinematical setups at the leptonic vertex. This simplifies calculations that are usually very time consuming.

\section{Coulomb Correction}

For the case of charged current processes, one of the leptons has electric charge and thus, besides the weak interaction, it would also interact with the nucleus via the static Coulomb interaction with the nucleus. This generally will distort the wave function of the charged lepton, that will not longer be accurately described by a free scattering state wave function (plane wave). One must however have in mind that this Coulomb correction of the electrons or muons involved in charged current scattering (or electron scattering) is just a technical issue that that can be exactly considered in a distorted wave Born approximation (DWBA) for the lepton, for instance in a partial wave expansion for the charged lepton $[12,13]$. 


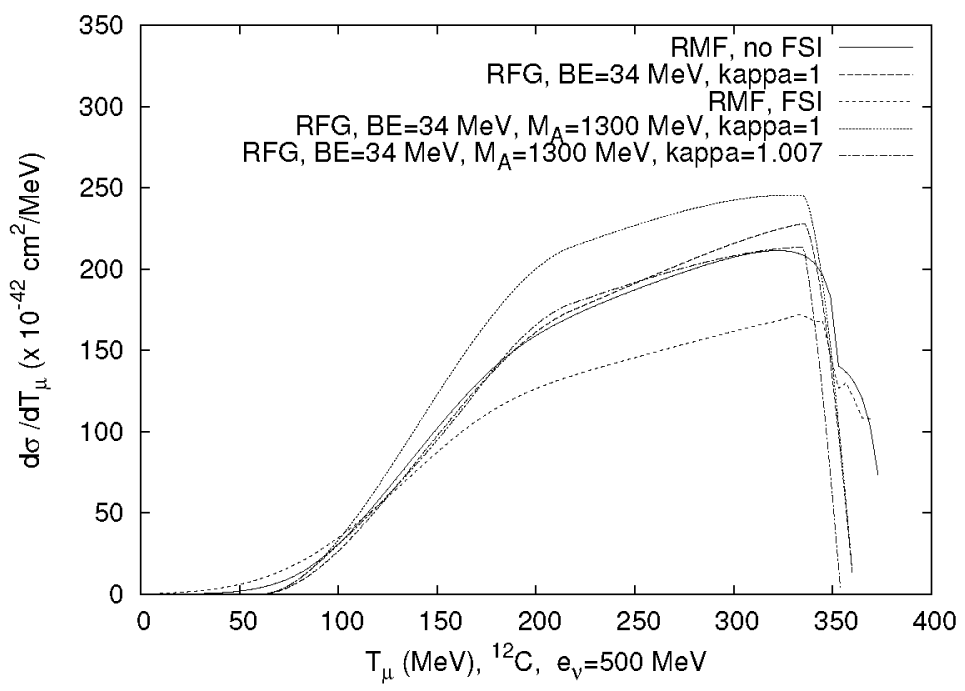

FIGURE 3. Predictions for muon neutrino cross-section on ${ }^{12} \mathrm{C}$, for a $500 \mathrm{MeV}$ muon neutrino beam. Results for a standard RFG calculation (dashed lines) are compared to RFG calculations with a modified value of the axial mass of $1300 \mathrm{MeV}$ (dotted lines), and with a further modification of the Pauli blocked values allowed for the knocked-out nucleon (dash-dotted curve). Further, the result of a relativistic shell model (RMF) calculation without (with) FSI is shown with solid (short dashed) curves

\section{Impulse Approximation and overlap integral}

Once the first order Born approximation, plus plane wave assumption for the leptons is assumed or PWBA, we now re-examine the structure of the hadronic vertex. As mentioned, a common approximation here is the Impulse Approximation (IA) one. This means considering that the virtual boson exchanged interacts mainly with only one nucleon, remaining the $A-1$ other nucleons as spectators in the reaction. The IA is a good approximation if the kinematics chosen for the reaction highly favours single nucleon interaction. This is the case for instance for quasielastic kinematics. We use the term quasielastic here as it is usually done in the context of electron scattering, that is, when the kinematics set by the lepton vertex is approximately the one required to scatter elastically a free nucleon. Further, the momentum transfer should have an associated wavelength of the order (or smaller) than the nucleon size. Thus a couple of hundreds of $\mathrm{MeV}$ of transferred momentum are required in order for the IA to work. Under these circumstances, IA amounts mainly to neglect the exchanged term where the virtual boson interacts also with nucleons from the spectator system. This is not favoured under quasielastic kinematics and many experiments with electrons show that the IA does a very decent work in describing quasi-elastic electron-nucleus scattering [12].

Under the IA, the matrix element of the current at the hadron vertex can be written as cast in Eq. (6), where $\hat{J}_{N}^{\mu}$ is an one-body operator and

$$
\psi_{\alpha}\left(\vec{r}_{1}\right) \sim \int d^{3} r_{2} \ldots d^{3} r_{A} \psi_{A-1, \alpha}^{+}\left(\vec{r}_{2} \ldots \vec{r}_{A}\right) \psi_{A}\left(\vec{r}_{1} \ldots \vec{r}_{A}\right)
$$

is the overlap integral. The index $\alpha$ means whatever quantum numbers and parameters are needed to fully specify the final state. Often it corresponds to hole-states of the initial nucleus, either discrete or in the continuum. Depending on the excitation energy allowed for the residual nucleus, a particular state or perhaps many of them will contribute to the observed cross-sections. In the Fermi Gas approach, this overlap will simply be given by the free nucleon wave function times the probability of finding a nucleon with a given energy and momentum in the initial nucleus. In a more realistic calculation, both the initial $A$-nucleon nucleus and the final $A-1$-one are described by shell model states, computed as Slater determinants made out of single-particle orbitals of the same mean field for the initial and final nuclei. Under this approach, the overlap will just collapse into the single-particle orbital for the hole nucleon, times the occupancy of said orbital. In this case, the 'norm' of the overlap will have the maximum possible value, usually referred as $100 \%$ of the shell model value. If the overlap is computed in a more realistic way, for instance if the initial and final Slater determinants are computed for different mean fields (as it should be, as they correspond to different nuclei), or if the initial and final nuclei are described by means of a general many body function beyond pure mean field Slater determinants, the norm of this overlap would be less than its corresponding value in the shell model, that 
is, it will be less than $100 \%$ of the shell model expectation. Norms of these overlaps (or spectroscopic factors) for selected states are obtained from nucleon knock-out experiments where the excitation energy of the residual system is fixed to pick-up of nucleons from particular single-particle states.

A few comments are in order. In Eq. (9), a further simplifying assumption has been made, namely the no presence of meson exchange currents. The importance of these MEC currents can be assessed by comparison of theory to electron-nucleus scattering data and, except at places where the IA contribution goes to zero, it is small for complex nuclei $(A>2)$ at quasielastic kinematics [9].

Thus, under all these assumptions, the nuclear many body physics remains contained into the overlap integral. Through this overlap, correlations and interactions among the many nucleons in the nuclear systems are considered. In the most general case, this overlap integral is a very complicated object, involving the degrees of freedom of $A-1$ nucleons and depending on the particular state of the residual system that is reached in the final state.

For light systems it can be computed from detailed microscopic calculations for selected final states of the residual system. For instance, the ${ }^{3} \mathrm{He}\left(e, e^{\prime} p\right) d$ reaction under quasielastic kinematics has been measured with high precision, and compared to detailed calculations that included the overlap the 3-body and 2-body initial and final nuclear systems, computed from realistic NN interactions plus variational or Faddeev calculations for the initial system. For the case of exclusive measurements, that is, when the final state is precisely known, these state of the art calculations should be very reliable and the comparison to exclusive experimental data for this reaction [14] shows that the IA does indeed an excellent job, provided that FSI are properly taken into account $[14,15]$.

\section{Relativistic Fermi Gas}

Within the relativistic Fermi gas, the nucleons are treated as free particles and thus to describe them, positive energy solutions of free Dirac equation are employed. The RFG, when provisions are made for binding energy corrections, can account reasonably well for inclusive QE electron scattering data by nuclei. Indeed, it has been shown that for inclusive results, that is for the results of several shells added together, there is little difference between RFG predictions and the ones where the nucleons are described by a mean field when FSI are not taken into account [16]. This can be seen here in Fig. 3. In this figure the cross-section for muon neutrino scattering off carbon is shown against the energy of the outgoing muon. This is an example of an inclusive measurement where one has to add up nucleons in the target nuclei, coming from all shells. The solid line, representing a relativistic shell model calculation and no FSI, yields very similar results to the standard RFG calculation (dashed lines), with parameters such as binding energy correction and Fermi momentum, tuned to reproduce electron scattering data.

\section{Final State Interaction}

Going one step further in the complexity of the model calculation, the final state interaction (FSI) of the knocked out nucleon with the residual system is taken into account. For inclusive scattering, a fair representation of FSI can be obtained by means of the same mean field employed to represent the target nuclei. When a relativistic representation of this mean field is employed (RMF), good agreement with data for inclusive electron scattering by nuclei at QE kinematics and intermediate energies $[10,16]$ is found. In Fig. 3 such calculation is depicted with short dashed lines showing a noticeable effect of FSI. Both the total cross-section as well as the shape of the cross-section change due to FSI. In said figure one can also see the effect of modifying parameters of the RFG calculation. In an attempt to fit the observed data by the MiniBooNE experiment [17], three parameters entering into the RFG calculation, namely the axial mass, the binding energy and a particular approach to Pauli blocking, were fit to data. One can see that the effect of increased axial mass (1300 MeV) gets compensated by the ad hoc enhancement of Pauli blocking (kappa=1.007, while kappa=1 means standard Pauli blocking) employed. One must keep in mind that the RFG is limited in the description of bound and scattered nucleons and it does not include FSI. While the RFG reproduces the bulk observations of electron-nucleus scattering, it cannot reproduce details of these cross-sections that the RMF model, that includes FSI, can easily accommodate. Thus it comes at no surprise that some tuning of the RFG parameters may be needed to reproduce MiniBooNE data, and this is a perfectly valid procedure to get more realistic outcome out of the RFG, only we should keep in mind that the resulting values of the parameters must be understood as effective values coming from the incorporation of FSI and other effects in the RFG. In other words, the axial mass needed to fit the experimental data of MiniBooNE with the RFG should not be compared to values of axial masses derived from other experiments. 
Coming back to the implementation of the final-state interactions, it has been achieved in different manners. In Ref. [18] a phenomenological convolution model was applied to the RFG, showing that nucleon re-scattering can produce a reduction of the quasi-elastic cross section as large as $15 \%$ at incoming neutrino energies of even about $1 \mathrm{GeV}$. A description of FSI mechanisms through the inclusion of relativistic optical potentials is presented in Refs. [16, 19, 20, 22, 23]. More specifically, Ref. [16] studies the uncertainties derived from the use of different prescriptions for the potentials. A reduction of the cross section of at least $14 \%$ is found at incoming neutrino energies of $1 \mathrm{GeV}$. In Refs. [22, 23], important FSI effects arise from the use of relativistic optical potentials within a relativistic Green's function approach.

Relativistic nuclear effects were included in the calculations of Refs. [16, 19, 20, 22, 23, 24, 25], using a relativistic shell model approach for the study of neutral-current and/or charged-current neutrino-nucleus scattering. In particular, in Refs. $[16,19,20]$ results in the relativistic plane-wave impulse approximation (RPWIA) were compared to RFG calculations. It is shown that binding-energy and shell effects tend to vanish, compared to RFG, as the energy increases, while FSI effects continue to make a sizeable effect with regard to RFG predictions, even at several GeV of neutrino energies.

Apart from relativistic dynamics and FSI, other effects may have an impact on neutrino-nucleus reactions. In Refs. $[26,27]$ the influence of relativistic nuclear structure effects, delta- and pion degrees-of-freedom, and RPA-type correlations on neutrino-scattering cross sections was examined. Ref. [28] includes long-range correlations, FSI and Coulomb corrections in ${ }^{12} \mathrm{C}\left(v_{\mu}, \mu^{-}\right){ }^{12} \mathrm{C}^{*}$ calculations. An alternative method was proposed in Ref. [29], where it was shown that a superscaling analysis of few-GeV inclusive electron scattering data allows one to predict charged-current neutrino cross sections in the nuclear resonance region, thereby effectively including delta isobar degrees-of-freedom.

\section{Factorization, spectral functions and scaling}

Under certain further assumptions to IA, a factorization prescription holds for the cross-sections we are computing,

so that it is possible to write them as the product of a kinematical factor, a nuclear spectral function $S\left(E_{m}, \vec{P}_{I}\right)$ independent of the reaction, and an elementary lepton-nucleon cross-section. The spectral function essentially contains the probability of having a nucleon in the target nucleus with determined missing energy and momentum. This spectral function is related to the overlap integral in Eq. (9). Under this factorization approach, the spectral function can be determined or fine-tuned by comparison to experiments with electrons, for instance, and applied to predict neutrinonucleus cross-sections. The hypothesis implied are OBE, IA, PWBA and factorization. If FSI are neglected, the spectral function will depend on two variables.

A further approach to lepton-nucleus scattering is the scaling one [30]. In order to avoid the nuclear uncertainties inherent in any neutrino-nucleus reaction model, several authors [30] have proposed to profit from the extensive knowledge on nuclear dynamics acquired from electron scattering experiments to predict inclusive charged-current (CC) and neutral-current (NC) neutrino-nucleus cross sections. The connection between the different electroweak processes is done by means of the superscaling analysis. In practice this goes beyond factorization as it further assumes that the nuclear response depends ultimately only on one scaling variable. The scaling approach has been shown to apply fairly well to inclusive electron scattering data, which allows to extract a scaling function, depending only on one scaling variable, from electron-nucleus scattering data. This scaling function can be applied to predict CC neutrinonucleus scattering and, under mild restrictions, also to NC neutrino-nucleus reactions, in the so-called Super Scaling (or SuSA) approach [30]. Incidentally, the results of the scaling approach are very similar to the ones obtained within the relativistic mean field (RMF).

\section{SUMMARY AND CONCLUSIONS}

The usual approximations required to compute neutrino-nucleus cross-section from theoretical models have been reviewed. These approximations (namely the Impulse Approximation) have been proved sensible when applied to QE electron scattering data and thus they should be considered reliable and safe to predict neutrino-nucleus scattering. They imply a sizeable effect of FSI in the cross-sections, even for neutrino energies of several thousands MeV. FSI are absent from RFG calculations, and thus it comes at no surprise the need for fine-tuning parameters of these RFG models in order to reproduce experimental data, likely incorporating in an effective way FSI effects. Further data with identification of the final knocked out proton or neutron, besides any possible charged lepton, will be extremely useful 
to constrain theoretical uncertainties.

\section{ACKNOWLEDGMENTS}

This work was partially supported by DGI (Spain) FPA2007-62216, FIS2008-04189, FPA2006-13807-C02-01, by the Junta de Andalucía, by the UCM and Comunidad de Madrid (Grupo de Física Nuclear, 910059), by the INFNMEC collaboration agreement "Study of relativistic dynamics in neutrino and electron scattering", and by the Spanish Consolider-Ingenio programme CPAN (CSD2007-00042).

\section{REFERENCES}

1. Y. Fukuda et al. (Super-Kamiokande Collaboration), Phys. Rev. Lett. 81, 1562 (1998); M.H. Ahn et al. (K2K Collaboration), Phys. Rev. Lett. 90, 041801 (2003); Q.-R. Ahmad et al. (SNO Collaboration), Phys. Rev. Lett. 87, 071301 (2001) and Phys. Rev. Lett. 89, 011301 (2002); K. Eguchi et al. (KamLAND Collaboration), Phys. Rev. Lett. 90, 021802 (2003); C. Athanassopoulos et al. (LSND Collaboration), Phys. Rev. Lett. 77, 3082 (1996) and Phys. Rev. Lett. 81, 1774 (1998).

2. Stuart J. Freedman and Boris Kayser, physics/0411216.

3. MINERvA Collaboration home page http://minerva.fnal.gov'

4. BooNE home page http://www-boone.fnal.gov/

5. NuTeV home page http://www-e815.fnal.gov/NuTeV.html

6. FINeSSE home page http://www-finesse.fnal.gov/

7. H. Saik et al. in "Technical Proposal for the Design, Construction, Commissioning and Operation of the ELISe setup", GSI, (2005).

8. T. Leitner, O. Buss, L. Alvarez-Ruso and U. Mosel, Phys. Rev. C 79034601 (2009); J. Lehr, M. Effenberger, U. Mosel, Nucl. Phys. A671, 503 (2000).

9. Y. Umino, J.M. Udias, Phys. Rev. C 52, 3399 (1995); Y. Umino, J.M. Udias, P.J. Mulders, Phys. Rev. Lett. 74, 4993 (1995).

10. M.C. Martinez, P. Lava, N. Jachowicz, J. Ryckebusch, K. Vantoumhout and J.M. Udias, Phys. Rev. C 73024607 (2006)

11. T. Donnelly, and A. Raskin, Amn. Phys. 167, 247 (1986).

12. J.M Udías, P. Sarriguren, E. Moya de Guerra, E. Garrido and J.A. Caballero, Phys. Rev. C 48, 2731 (1993).

13. J.M. Udías, P. Sarriguren, E. Moya de Guerra and J.A. Caballero, Phys. Rev. C 53, R1488 (1996).

14. M. M. Rvachev et al., Phys. Rev. Lett. 94, 192302 (2005).

15. Javier R. Vignote, R. Alvarez-Rodriguez, C. Fernandez-Ramirez, E. Garrido, E. Moya de Guerra, P. Sarriguren and J.M. Udias, in Nuclear Theory 23, "Proceedings of the XXV International Workshop on Nuclear Physics", Rila Mountains, Heron Press, S. Dimitrova ed., (2004), 85-98.

16. C. Maieron, M.C. Martínez, J.A. Caballero, and J.M. Udías, Phys. Rev. C 68, 048501 (2003).

17. A. A. Aguilar-Arevalo et al., Phys. Rev. Lett. 100, 032301 (2008).

18. C. Bleve, G. Co, I. De Mitri, P. Bemardini, G. Mancarella, D. Martello and A. Surdo, Astropart. Phys. 16, 145 (2001).

19. W.M. Alberico, M.B. Barbaro, S.M. Bilenky, J.A. Caballero, C. Giunti, et al., Nucl. Phys. A623, 471 (1997).

20. W.M. Alberico, M.B. Barbaro, S.M. Bilenky, J.A. Caballero, C. Giunti, et al., Phys. Lett. B 438, 9 (1998).

21. C. Praet, N. Jachowicz, J. Ryckebusch, P. Vancraeyveld, K. Vantournhout, Phys. Rev. C 74, 065501 (2006).

22. A. Meucci, C. Giusti, and F.D. Pacati, Nucl. Phys. A744, 307 (2004).

23. A. Meucci, C. Giusti, and F.D. Pacati, Nucl. Phys. A739, 277 (2004).

24. M.B. Barbaro, A. De pace, T.W. Donnelly, A. Molinari, and M.J. Musolf, Phys. Rev. C 54, 1954 (1996).

25. B.I.S. van der Ventel and J. Piekarewicz, Phys. Rev. C 69, 035501 (2004).

26. H. Kim, J. Piekarewicz, and C.J. Horowitz, Phys. Rev. C 51, 2739 (1995).

27. H. Kim, S. Schramm, and C.J. Horowitz, Phys. Rev. C 53, 2468 (1996);53, 3131 (1996).

28. J. Nieves, J.E. Amaro, and M. Valverde, Phys. Rev. C 70, 055503 (2004).

29. J.E. Amaro, M.B. Barbaro, J.A. Caballero, T.W. Donnelly, A. Molinari, and I. Sick, Phys. Rev. C 71, 015501 (2005).

30. M.C. Martinez, J.A. Caballero, T.W. Donnelly, J.M. Udias, Phys. Rev. C77, 064604 (2008), Phys. Rev.Lett. 100, 052502 (2008); J.A. Caballero, J.E. Amaro, M.B. Barbaro, T.W. Donnelly, J.M. Udias, Phys. Lett. B653, 366-372 (2007); J.E. Amaro, M.B. Barbaro, J.A. Caballero, T.W. Donnelly, J.M. Udias, Phys. Rev. C 75, 034613 (2007); J.A. Caballero, J.E. Amaro, M.B. Barbaro, T.W. Donnelly, C. Maieron, J.M. Udias, Phys. Rev. Lett.95, 252502 (2005). 